\title{
Implicit probabilistic sequence learning is independent of explicit awareness
}

\author{
Sunbin Song, ${ }^{1,2,5}$ James H. Howard Jr., ${ }^{3,4}$ and Darlene V. Howard ${ }^{1,2}$ \\ ${ }^{1}$ Department of Psychology, Georgetown University, Washington, District of Columbia 20057, USA; ${ }^{2}$ Interdisciplinary Program \\ in Neuroscience, Georgetown University, Washington, District of Columbia 20057, USA; ${ }^{3}$ Department of Neurology, \\ Georgetown University, Washington, District of Columbia 20057, USA; ${ }^{4}$ Department of Psychology, Catholic University, \\ Washington, District of Columbia 20064, USA
}

\begin{abstract}
Studies into interactions between explicit and implicit motor sequence learning have yielded mixed results. Some of these discrepancies have been attributed to difficulties in isolating implicit learning. In the present study, the effect of explicit knowledge on implicit learning was investigated using a modified version of the Alternating Serial Response Time (ASRT) task, a probabilistic sequence learning paradigm that yields continuous and relatively pure measures of implicit learning. Results revealed that implicit learning occurred to the same extent, whether or not subjects had explicit knowledge. Some evidence, however, indicated that explicit knowledge could interfere with the expression of implicit learning early in training. In addition, there were dissociations between learning measures, in that reaction time and accuracy were differentially affected by explicit knowledge. These findings indicate that implicit sequence learning occurs independently of explicit knowledge, and help to explain previous discrepant findings.
\end{abstract}

Though implicit sequence learning has been extensively studied, less is known about the interaction between explicit and implicit learning. Implicit learning occurs incidentally in the absence of conscious knowledge, and it differs from explicit learning, which is conscious and intentional (Frensch 1998). The Serial Response Time Task (SRTT) has been used extensively to study implicit sequence learning, but has disadvantages in that explicit learning of the sequence often occurs with longer training (Willingham 2001). In the SRTT, subjects typically watch one of four dots light up in a predetermined order and respond by pressing the corresponding button. Learning is shown by faster and more accurate performance on these patterned blocks (fixed deterministic sequence) as compared with a block of random trials (Nissen and Bullemer 1987).

Sequence learning has also been exhibited for probabilistic sequences in which certain sequences of trials occur more frequently than others but do not follow a fixed sequence of events (Schvaneveldt and Gomez 1998; Jimenez and Mendez 1999; Howard et al. 2004a; Jimenez et al. 2006). For example, in the Alternating Serial Response Time Task (ASRT) used in the present experiment, Pattern and Random trials alternate (e.g., 1r4r3r2r, where the numbers refer to positions $1,2,3$, and 4 , and "r" refers to a randomly chosen one of these four locations). This structure causes certain triplets of trials (e.g., 134, 423) to occur at a higher frequency than others (e.g., 132, 422). Subjects become faster and more accurate for High-frequency compared with Low-frequency triplets (Howard et al. 2004a).

Imaging studies have shown that sequence learning depends on frontal-striatal circuits (Barone and Joseph 1989; Rauch et al. 1997; Exner et al. 2002; Destrebecqz et al. 2005; Aizenstein et al. 2006; Ashe et al. 2006; Histed and Miller 2006), making it useful for the study of disorders such as schizophrenia (Weickert et al. 2002; Schwartz et al. 2003), OCD (Kathmann et al. 2005), Parkinson's disease (Smith and McDowall 2004), mild cognitive impairment (Negash et al. 2006), and dyslexia (Howard et al. 2006).

\section{${ }^{5}$ Corresponding author.}

E-mail sss35@georgetown.edu; fax (202) 687-6050.

Article is online at http://www.learnmem.org/cgi/doi/10.1101//m.437407.
In addition, by giving instructions, this task can be made into an explicit sequence learning paradigm, making it useful for the study of explicit/implicit interactions. These studies, however, have yielded conflicting results. In one imaging study of young adults, it was shown that explicit learning interfered with implicit learning for complex (ASRT) but not simple deterministic (SRTT) sequences (Fletcher et al. 2004). Imaging results suggested that competition for frontal lobe resources accounted for these results for complex sequences. In a behavioral study using the ASRT, it was found in young subjects that these two processes did not interfere with each other, but they did for elderly subjects (Howard and Howard 2001). Other imaging studies using the SRTT paradigm suggested that even for deterministic sequences, implicit and explicit learning were not occurring simultaneously, as there was little overlap between brain activation patterns for these two conditions (Grafton et al. 1995; Rauch et al. 1995; Hazeltine et al. 1997). However, Willingham and GoedertEschmann (1999) found behaviorally that implicit learning occurred to the same extent for implicit and explicit conditions, suggesting simultaneous processes.

A subsequent imaging study supported these behavioral findings in that similar brain systems were activated in both the implicit and explicit condition (Willingham et al. 2002). In Willingham's studies, subjects in the explicit group were told that a color cue would be present to alert them when the patterned blocks were occurring, and would be absent for random blocks. Unbeknownst to these subjects, however, the patterned blocks were often uncued, leading to an explicit covert condition. The reaction time difference between random and pattern blocks in this explicit covert condition was identical to that in the implicit learning group, consistent with Willingham's COBALT theory (Willingham and Goedert-Eschmann 1999) that implicit and explicit processes occur in parallel as long as a motor response is being made.

Some of these discrepancies concerning the relation between implicit and explicit learning may arise from differences in sequence complexity and in the presence of explicit awareness even in the implicit group. Since explicit awareness is usually gained with extended training in the SRTT, there is controversy as to whether or not pure implicit learning even occurs (Shanks 
2005). Dual task conditions are often needed to prevent explicit awareness, but these manipulations add other variables that can change the pattern of results (Cohen et al. 1990; Curran and Keele 1993; Frensch et al. 1994; Stadler 1995; Shanks and Channon 2002).

In the study of explicit/implicit interaction, probabilistic sequence learning paradigms like the ASRT may reveal insights not previously shown in studies using deterministic SRTT paradigms. First, in the group given incidental instruction (no explicit mention of the pattern), the sequence is harder to discover in probabilistic sequences minimizing the contamination of implicit learning by explicit knowledge (Schvaneveldt and Gomez 1998; Jimenez and Mendez 1999; Howard et al. 2004a; Jimenez et al. 2006). This also allows for more extensive training than the SRTT. For example, using the ASRT, subjects were given 10 separate sessions of training during which they encountered 2100 repetitions of the pattern, and still they revealed no awareness of the sequence as assessed by verbal report and by a sensitive generation test (Howard et al. 2004a). Second, sequences in probabilistic paradigms are usually complex, in that for the ASRT, the lowest level of regularity is second-order (integration is required across at least three trials) (Howard and Howard 1997). In contrast, differing levels of complexity on SRTT paradigms may lead to differing results for explicit/implicit interactions (Cohen et al. 1990; Frensch et al. 1994). Third, for deterministic sequences, blocks of trials are assessed, making it difficult to parse out the various stages of learning (Halsband and Lange 2006). In contrast, learning measures for probabilistic sequences are continuously available, because less predictable trials can be compared with more predictable ones throughout, and require no change in the structure of the practiced sequence. Finally, the higher error rate that typically occurs for probabilistic sequences allows for errors to be examined in addition to reaction time (Schvaneveldt and Gomez 1998; Jimenez and Mendez 1999; Howard et al. 2004a).

In the present study, we used a modified version of the ASRT to explore the relation between explicit knowledge and implicit learning, comparing people who were told about the presence of the regularity (Intentional instructions) with those who were not (Incidental instructions). Due to the implicit nature of the task, even with instruction, awareness is hard to obtain (Howard and Howard 2001). Thus, in the version used here, for both instructional groups color cues (gray or red) were added to Pattern trials in most blocks to facilitate explicit awareness for people given Intentional instructions (Cued blocks). On other blocks, these color cues were removed in order to eliminate the contributions of explicit knowledge, even though the sequence pattern was still present. These Probe blocks were inserted to allow for implicit learning measures to be obtained in subjects with explicit knowledge of the pattern. Similar to Willingham et al. (2002), we reasoned that if implicit learning was affected by explicit knowledge, learning measures on these Probe blocks would differ based on instruction. However, if implicit learning occurred independently of explicit knowledge, then implicit learning on Probe blocks would be the same for Incidental and Intentional subjects.

There is also the question of how to measure the extent to which explicit knowledge is used. In SRTT paradigms, explicit awareness typically speeds subjects' responses (Willingham and Goedert-Eschmann 1999). It is clear that someone has explicit knowledge if they can accurately report a significant portion of the pattern. However, there is no way to judge to what degree the subject has chosen to use such explicit knowledge to speed their responses, since there is no basis for comparison. In the present study, we can measure both the acquisition of explicit knowledge (by the ability to accurately report the pattern) and the use of explicit knowledge, as we can compare reaction times for High- frequency trials under conditions in which explicit knowledge can or cannot be used.

In addition, due to continuous measures for both reaction time and accuracy, by comparing Cued and Probe blocks we can observe the effects of explicit knowledge on reaction time versus accuracy learning measures. A dissociation between accuracy and reaction time has been found in several other paradigms in which accuracy offered a reliable measure of learning (Hikosaka et al. 1995, 2002; Miyachi et al. 1997; Sakai et al. 1998; Rand et al. 2000; Watanabe et al. 2006). These studies showed different effects of bimanual transfer, retention, explicit transfer, and dual task on these two measures. Therefore, we hypothesized that explicit awareness would also differentially affect accuracy and reaction time, which would support claims that they depend on two independent sequence learning systems (Watanabe et al. 2006).

\section{Results}

In the ASRT, predictable Pattern trials alternate with unpredictable Random ones, causing certain triplets of trials to occur at a higher frequency than others. For example, for the pattern 1r3r2r4r, triplets beginning with 1 and ending with 3 are Highfrequency, whereas those beginning with 3 and ending with 1 are Low-frequency. Performance is better on the third event if it falls on a High-frequency triplet versus a Low-frequency triplet, regardless of whether this High-frequency triplet falls on a Pattern or Random trial (Howard et al. 2004a). Pattern trials are always High-frequency, whereas one-fourth of Random trials are Highfrequency by chance, such that 16 High-frequency triplets occur $62.5 \%$ of the time and 32 Low-frequency triplets (excluding repetitions and trills as defined below) occur $25 \%$ of the time. Each High-frequency triplet occurs on average five times more often than a Low-frequency triplet. Previous studies have shown that these triplet frequencies are learned implicitly, and this is revealed in reaction time and accuracy, with differences between High and Low triplet sequences (triplet type effect) increasing with practice (Howard et al. 2004a).

For all results reported in the following sections, High- and Low-frequency triplets are compared. Trills (i.e., 121) and repetitions (i.e., 111) are removed from these analyses because they are not counterbalanced across subjects, in that they are always Lowfrequency for all subjects. Thus, performance on trills and repetitions could reflect preexisting biases, rather than sequence specific learning.

The color of the cue was found to have no effect on any of the measures. Thus, data from both red and gray cue groups were combined for all the analyses.

\section{Explicit awareness measures}

For subjects given Intentional instructions, a sequence description task occurred after each block. Explicit knowledge was said to have been attained at the first block at which the subject guessed the correct pattern, and continued to repeat the correct pattern throughout the sessions. On this sequence description task, by this criterion, all of the 12 subjects given Intentional instructions gained full explicit knowledge of the pattern within the first two epochs (one epoch is five blocks), and 11 of 12 subjects gained full knowledge within the first epoch. Complete explicit knowledge of the pattern sequence was reached on average on block $3 \pm 2$ (standard deviation) of the first epoch, i.e., after $\sim 30$ repetitions of the pattern. (Across all sessions, subjects completed 450 repetitions of the pattern.)

On a generation task presented after the ASRT, half of the subjects given Intentional instructions were able to perfectly pro-

\section{Learning \& Memory}


duce the correct pattern from the Cued trials, whereas no subject given Incidental instructions could do so. For Intentional group subjects not able to generate the pattern, inquiry revealed that they did not understand that generation was for Cued blocks only. Therefore, for future studies, subjects should be asked to separately generate the pattern for both the Cued (alternating colors) and the Probe (all black) blocks.

Regarding answers to questions about the Cued epochs in an end-of-experiment interview, all Intentional subjects reported knowledge of the alternating regularity on Cued blocks, whereas no Incidental subjects did so. For questions concerning the Probe blocks, no one, irrespective of instruction, reported awareness of the correct pattern on Probe blocks, demonstrating that learning exhibited during these blocks reflected implicit learning in both groups.

A card sorting task given at the end also revealed that learning was implicit during Probe epochs. The triplets on each card of the sorting task were filled in with black, corresponding to the Probe epochs at the end of each session. The percentage of Highand Low-frequency cards sorted correctly was not significantly different from chance in either group (Incidental Mean $47.4 \%$, SD $6.5 \%, t=-1.5, P=0.2$, Intentional Mean 54.6\%, SD $11 \%$, $\mathrm{t}=1.4, P=0.2)$.

In summary, (1) Incidental subjects showed no knowledge of the regularity by any of these explicit measures, and (2) Intentional subjects did gain explicit knowledge for the Cued alternating color blocks within the first epoch of training, and yet showed no evidence of being aware that the pattern was present on the all-black Probe ones.

\section{Reaction time measures}

Median Reaction Times (for correct trials only) were determined separately for the third event of High-frequency versus Lowfrequency triplet types for each block for each instruction group. The means of these median reaction times across epochs for High- and Low-frequency triplets are shown in Figure 1, with Figure 1A depicting the Intentional and Figure $1 \mathrm{~B}$ the Incidental group. The all-black Probe epochs 3, 6, and 9 are indicated as such along the $X$-axis.

A mixed design instruction (Incidental vs. Intentional) by epoch (1-9) by triplet type (High vs. Low) ANOVA of reaction time revealed significant main effects of epoch $\left(F_{(8,176)}=33.6\right.$, MSE $=479.7, P<0.001)$, indicating that people got faster with practice, and triplet type $\left(F_{(1,22)}=52.0, \mathrm{MSE}=1194.9, P<0.001\right)$, showing that High-frequency triplets were faster than Lowfrequency ones. There was also an epoch by triplet type interaction $\left(F_{(8,176)}=3.5\right.$, MSE $\left.=153.6, P<0.001\right)$, indicating that this High/Low difference changed with practice and therefore that sequence learning had occurred. There was no main effect of instruction, indicating that the instructional groups did not differ in overall reaction time. Most importantly, there was a significant instruction by epoch by triplet type interaction $\left(F_{(8,176)}=2.5, \mathrm{MSE}=153.6, P<0.02\right)$.

To examine this interaction further, we plotted the High versus Low triplet type effect (i.e., RT on Low-frequency triplets minus RT on High-frequency triplets) for each group and epoch (Figure 2). The Cued and the Probe epochs were submitted to separate instruction by triplet type (High vs. Low) by epoch ANOVAs. For both Cued and Probe epoch analyses, there was an epoch by triplet type interaction $\left(F_{(5,110)}=3.6, \mathrm{MSE}=122.3\right.$, $\left.p<0.005 ; F_{(2,44)}=5.1, \mathrm{MSE}=89.1, P<0.02\right)$, indicating that learning as measured by the triplet type effect occurred across both types of blocks.

In the Cued epochs, our explicit measures had indicated that the Incidental group relied only on implicit learning, whereas the Intentional group relied on both implicit learning and explicit knowledge. The instructional groups differed on Cued epochs, suggesting that explicit knowledge could be used to increase the triplet type effect in reaction time. This is apparent both in Figure 2 and in the ANOVA for Cued epochs, which revealed a trend for a triplet type by instruction interaction $\left(F_{(1,22)}=3.9, \mathrm{MSE}=1514.5, P<0.061\right)$. In contrast, the instructional groups did not differ from each other on the Probe epochs $(3,6$, and 9), which according to our explicit measures above are tapping implicit learning in both groups. This is apparent both in the figure and in the ANOVA for Probe epochs, where no main effects or interactions with instruction approached significance; triplet type by instruction $F_{(1,22)}=0.6, \mathrm{MSE}=119.0, P<0.44$. This pattern suggests that implicit learning is unaffected by explicit knowledge.

We then conducted analyses using only High-frequency triplets. The goal was to provide a different test of the conclusion

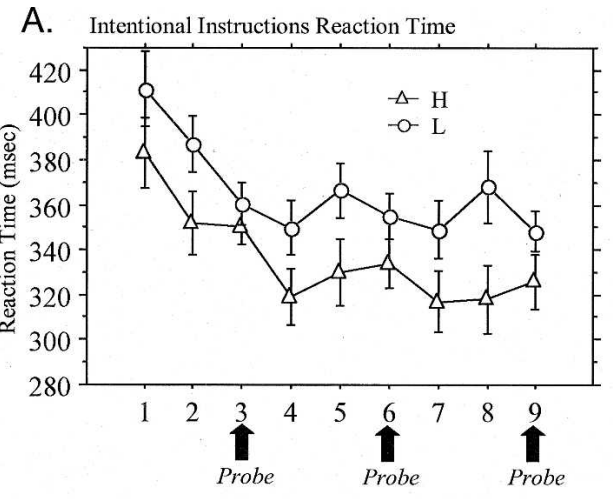

Epoch

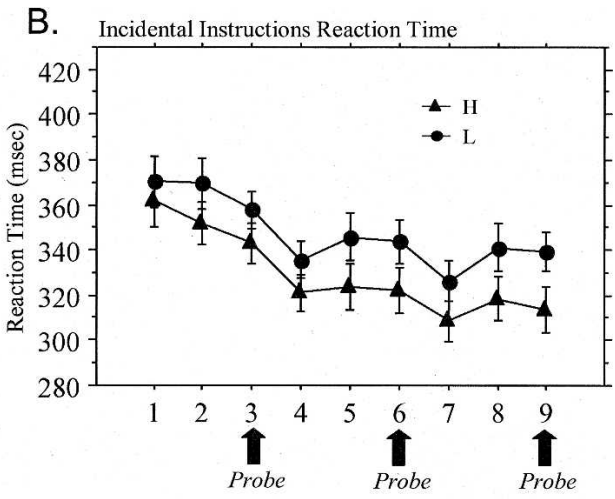

Epoch

Figure 1. Reaction time measures for Intentional and Incidental instruction groups. ( $A$ ) Mean of median reaction times for subjects given Intentional instructions $(n=12)$ split by High- $(\triangle)$ and Low- $(O)$ frequency triplets. $(B)$ Mean of median reaction times for subjects given Incidental instructions $(n=12)$ split by High- $(\boldsymbol{\Delta})$ and Low- $(\bullet)$ frequency triplets. (Arrows) Probe epochs $(3,6,9)$. A mixed design instruction (Incidental vs. Intentional) by epoch (1-9) by triplet type (High vs. Low) ANOVA of reaction time revealed significant main effects of epoch $\left(F_{(8,176)}=33.6, \mathrm{MSE}=479.7, P<0.001\right)$, indicating that people became faster with practice, and triplet type $\left(F_{(1,22)}=52.0, \mathrm{MSE}=1194.9, P<0.001\right)$, showing that High-frequency triplets were faster than Low-frequency ones. There was also an epoch by triplet type interaction $\left(F_{(8,176)}=3.5, \mathrm{MSE}=153.6, P<0.001\right)$, indicating that this High/Low difference changed with practice, and therefore sequence learning had occurred. There was no main effect of instruction, indicating that the instructional groups did not differ in overall reaction time. Most importantly, there was a significant instruction by epoch by triplet type interaction $\left(F_{(8,176)}=2.5, \mathrm{MSE}=153.6, P<0.02\right)$. 
that all epochs for the Incidental group, and the Probe epochs for the Intentional group, are reflecting only implicit learning, whereas the Cued epochs in the Intentional group reflect both implicit learning and explicit knowledge. These analyses take advantage of the fact that although Pattern events are always Highfrequency triplet trials, Random events may by chance be Highfrequency triplet trials $25 \%$ of the time. Previous experiments have shown that when the triplet frequency is learned implicitly, Pattern- and Random-High-frequency triplet trials do not differ either in reaction time or accuracy, though both of these trial types differ significantly from Low-frequency triplet trials (Howard et al. 2004a). However, in contrast with implicit learning, any explicit knowledge of the alternating rule should result in people differentiating between Pattern and Random events even when both are High-frequency.

Therefore, if we are correct that explicit knowledge is being used only by Intentional subjects and only during Cued epochs, then on Probe epochs there should be no difference between Pattern and Random events within High-frequency trials for either group, because neither group is using explicit knowledge. However, on Cued epochs when the Intentional, but not the Incidental, group is calling on explicit knowledge, the groups should differ on this Pattern versus Random measure.

To test this prediction, reaction time difference measures for Random-High- minus Pattern-High-frequency triplet trials only are plotted in Figure 3. A mixed design instruction (Incidental vs. Intentional) by epoch (1-9) by event type (Random-High vs. Pattern-High) ANOVA on these data revealed a significant event type by epoch by instruction interaction $\left(F_{(8,176)}=2.7\right.$, MSE $=535.6, P<0.02$ ). The Cued and the Probe epochs were then submitted to separate instruction by event type (RandomHigh vs. Pattern-High) by epoch ANOVAs. For Probe blocks, there was no event type by instruction interaction $\left(F_{(1,22)}=0.4\right.$, MSE $=90.0, P<0.6$ ), whereas for Cued blocks, there was a significant interaction $\left(F_{(1,22)}=5.1, \mathrm{MSE}=2349.7, P<0.04\right)$. This supports the hypothesis that on Cued epochs, explicit knowledge affected reaction time on Pattern versus Random events for Intentional subjects, but did not have any such effect on Probe epochs.

One puzzling aspect of the data in Figure 3 is that in the initial session (epochs 1-3), the Random-High trials were actually

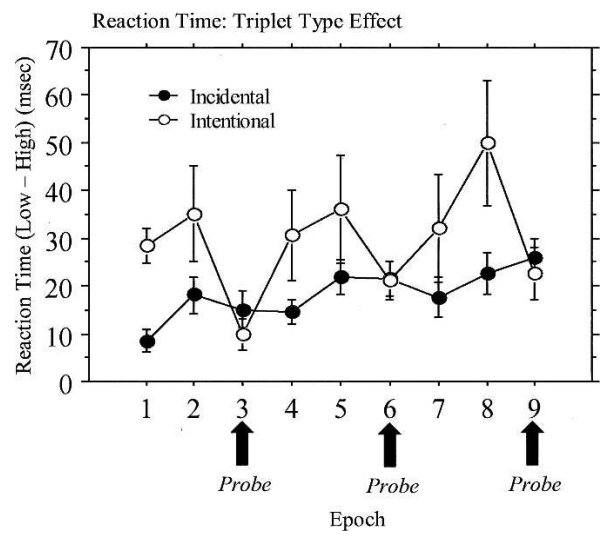

Figure 2. Overall, implicit learning proceeds unaffected by explicit knowledge. Reaction Time triplet type effects (RT for Low-frequency minus RT for High-frequency triplets) are plotted for Incidental $(\bullet)$ and Intentional $(O)$ instruction groups. An ANOVA for Cued epochs revealed a trend for a triplet type by instruction interaction $\left(F_{(1,22)}=3.9\right.$, $\mathrm{MSE}=1514.5, P<0.061)$. In contrast, the instructional groups did not differ from each other on the Probe epochs (3, 6, and 9). For Probe epochs, no main effects or interactions with instruction approached significance; triplet type by instruction $F_{(8,176)}=0.6, \mathrm{MSE}=119.0, P<0.44$.

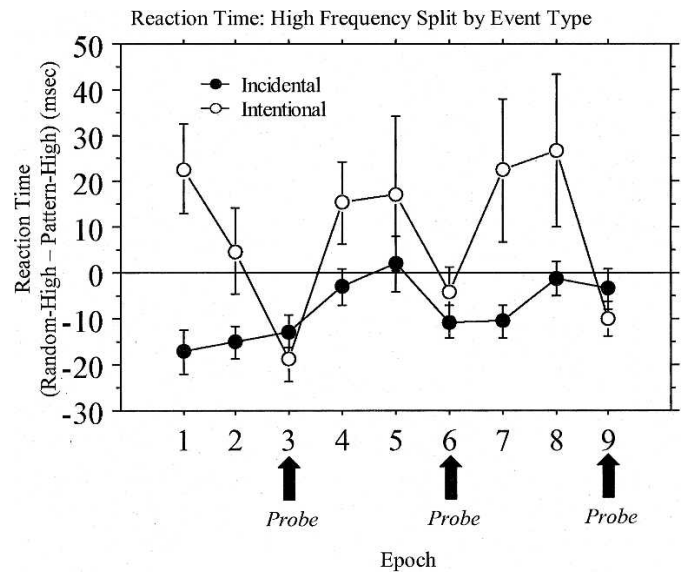

Figure 3. Explicit knowledge can affect Reaction Time on Highfrequency (Pattern vs. Random) events. Reaction Time event type effects are plotted (RT for Random-High-frequency minus RT for Pattern-Highfrequency triplets) for both Incidental $(\bullet)$ and Intentional $(O)$ instruction groups. A mixed-design instruction (Incidental vs. Intentional) by epoch (1-9) by event type (Random-High vs. Pattern-High) ANOVA on these data revealed only a significant event type by epoch by instruction interaction $\left(F_{(8,176)}=2.7, \mathrm{MSE}=535.6 P<0.02\right)$. The Cued and the Probe epochs were then submitted to separate instruction by event type (Random-High vs. Pattern-High) by epoch ANOVAs. For Probe blocks, there was no event type by instruction interaction, whereas for Cued blocks, there was a significant interaction $\left(F_{(1,22)}=5.1, \mathrm{MSE}=2349.7, P<0.04\right)$.

faster than the Pattern-High trials for Incidental subjects (and Intentional subjects in Probe epoch 3). This difference was present from the very first block, but disappeared by the second session. This pattern was also consistent across subjects. Further analysis ${ }^{6}$ showed that this difference reflected pre-existing sequential effects due to certain structural regularities beyond three trials. Such sequential effects, which have been reported in previous research (for example, see Soetens et al. 2004), are overcome with training.

\section{Accuracy measures}

All of the above analyses were also conducted on accuracy. Proportion correct for High- and Low-frequency triplets for both instruction groups is shown in Figure 4. A mixed design instruction (Incidental vs. Intentional) by epoch (1-9) by triplet type (High vs. Low) ANOVA of accuracy revealed significant main effects of epoch $\left(F_{(8,176)}=2.8\right.$, MSE $\left.=0.002, P<0.007\right)$ as accuracy decreased over time. Figure 4 indicates that this is due to falling accuracy for Low-frequency triplets, while accuracy for High-frequency triplets remained stable. This is confirmed by a significant effect of triplet type $\left(F_{(1,22)}=92.7, \mathrm{MSE}=0.003\right.$, $P<0.001)$ as well as an epoch by triplet type interaction

${ }^{6}$ Though Random-High and Pattern-High trials are identical in terms of triplet frequency, since Random-High trials always follow Pattern-High trials, they are not identical in terms of quad sequence (four successive elements) or higher structure. Low-frequency triplets also differ in terms of quad structure. Local sequential regularities of this sort have been shown to influence RT in previous sequence learning tasks (Soetens et al. 2004). For example, in the present study, quad types can be split into those that contain two repeated pairs (i.e. 1122); a repeated pair in the first (1124), second (1224), or last (1244) position; a run of three in the first position (1112); a trill in the first position (1213); or no repeated elements (1243). Pattern-High trials can occur within all of these quad types, but Random-High trials do not. Similarly, Low-frequency trials do not occur on quads that began with trills or repetitions, nor did they occur on quads with two repeated elements. When all unequally represented quad types of this sort were removed to eliminate local sequential effects, the difference between the Pattern-High and Random-High trials seen in the first session disappeared, whereas the difference between the Low- and both Highfrequency trial types remained. 
A. Intentional Instructions Accuracy

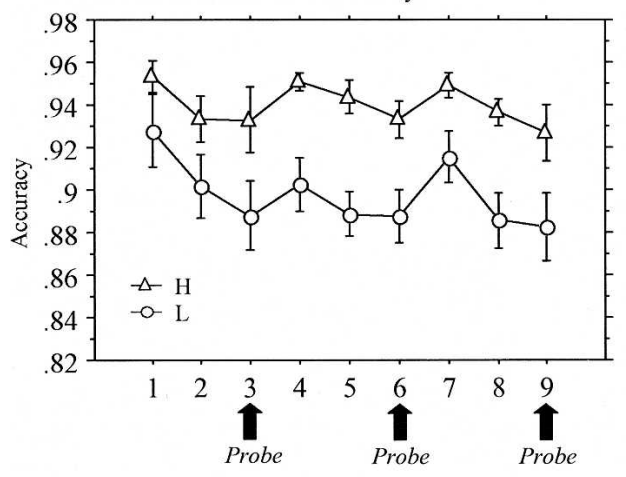

Epoch
B.

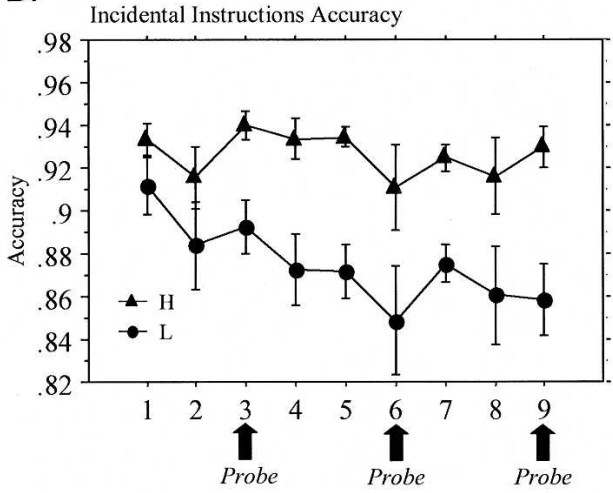

Figure 4. Accuracy measures for Intentional and Incidental instruction groups. (A) Percent correct for subjects given Intentional instructions are plotted for High- $(\triangle)$ versus Low- $(O)$ frequency triplets. $(B)$ Percent correct for subjects given Incidental instructions are plotted for High- $(\boldsymbol{\Lambda})$ versus Low- $(\bullet)$ frequency triplets. Learning revealed by a significant effect of triplet type $\left(F_{(1,22)}=92.7, \mathrm{MSE}=0.003, P<0.001\right)$ as well as an epoch by triplet type interaction $\left(F_{(8,176)}=2.4, \mathrm{MSE}=0.001, P<0.02\right)$. No main effects or interactions with instruction were significant.

$\left(F_{(8,176)}=2.4, \mathrm{MSE}=0.001, P<0.02\right)$. No main effects or interactions with instruction approached significance.

In particular, unlike reaction time, there was no epoch by triplet type by instruction interaction $\left(F_{(8,176)}=0.4\right.$, MSE $=0.001$, $P<0.95)$. This can be seen more clearly in Figure 5, where triplet type effects for accuracy are plotted. In the Intentional group, accuracy was not affected by explicit knowledge on Cued blocks as compared with Probe blocks, and the Instructional groups do not differ significantly in the magnitude of the triplet type effect. Therefore, explicit knowledge is not influencing the magnitude of the triplet type effect for the accuracy measure on Cued blocks, even though it had for the Reaction Time measure.

This conclusion is supported further by Figure 6, in which only High-frequency triplet trials are plotted. In the reaction time measures (Fig. 3), the presence of explicit knowledge in the Intentional group led to a significant difference between Pattern-

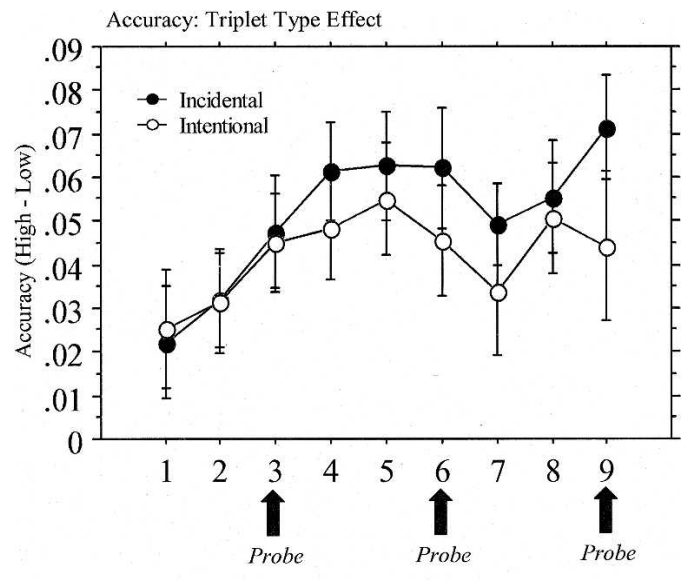

Epoch

Figure 5. Accuracy is not affected by explicit knowledge. Accuracy triplet type effects (accuracy for High-frequency minus accuracy for Lowfrequency triplets) are plotted for Incidental $(\bullet)$ and Intentional $(\bigcirc)$ subjects. Unlike reaction time, accuracy was not affected by explicit knowledge on Cued blocks as compared with Probe blocks, and the Instructional groups do not differ significantly in the magnitude of the triplet type effect. Therefore, explicit knowledge is not influencing the magnitude of the triplet type effect for the accuracy measure, even though it had for the RT measure.
High and Random-High events on cued epochs. In contrast, Figure 6 shows that for accuracy there were no significant differences between Pattern-High and Random-High events for either group.

Accuracy and Reaction Time measures thus seem to reflect different components of learning in this task; triplet type (High vs. Low) and event type (Pattern-High vs. Random-High) effects were both influenced by explicit knowledge on cued blocks, but only when measured via reaction time, not via accuracy.

\section{Correlations}

Though there were no significant effects of explicit knowledge on implicit learning measures on Probe blocks in the above analyses, the standard error bars in Figures 2 and 3 suggest that there was large variation in the magnitude of the triplet type and event type effects on Cued epochs within the Intentional group. This variability does not reflect differences in the extent to which Intentional subjects have explicit knowledge of the sequence, because, as reported above, by the end of the first epoch 11 of 12 Intentional subjects were able to report the regularity perfectly. Therefore, this variability suggests that the Intentional subjects vary in the extent to which they used explicit knowledge to speed their responses during Cued epochs.

To find out whether using explicit knowledge influenced measures of implicit learning in the Intentional group, we performed correlations for reaction time measures for the Intentional group. The extent to which the person used explicit knowledge was assessed via the Random-High minus PatternHigh Reaction Time difference in the second epoch (which was Cued) of a given session. The measure of implicit learning was the Low-Frequency-High-Frequency Reaction Time difference in the third epoch of a given session, which was a Probe epoch. For the first session, this correlation was negative and significant, $r(10)=-0.66, P<0.02$, indicating that the more Intentional subjects used their explicit knowledge during Cued epoch 2, the less implicit learning they showed on Probe epoch 3. The resulting scatterplot is shown in Figure 7. This effect disappeared by the second (comparing the fifth and sixth epochs) and third (comparing the eighth and ninth epochs) sessions; for the second session, $r(10)=0.25, P<0.44$, and for the third session, $r(10)=0.26, P<0.41$. When we performed these correlations in the Incidental group, they were not significant for any of the sessions.

This first session effect for the Intentional group could not 


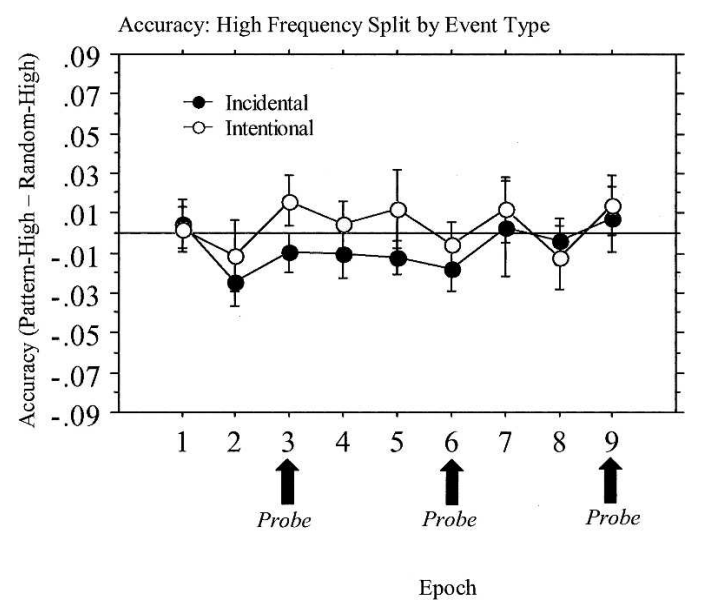

Figure 6. Unlike Reaction Time, accuracy measures do not reflect the use of explicit knowledge. Accuracy event type effects (ACC for PatternHigh minus ACC for Random-High-frequency triplets) are plotted for both Incidental $(\bullet)$ and Intentional $(\bigcirc)$ instruction groups. In the reaction time measures (Fig. 3), the presence of explicit knowledge in the Intentonal group led to a significant difference between Pattern-High and Random-High events on cued epochs. In contrast, for accuracy there were no significant differences between Pattern-High and Random-High events for either group.

be accounted for by when the subject gained explicit awareness; the correlation between the block on which awareness was gained and implicit learning in epoch 3 (Low-Frequency-HighFrequency Reaction Time) was not significant, $r(10)=-0.14$, $P<0.66)$.

It seemed possible that the negative correlation between use of explicit knowledge and the amount of implicit learning revealed during the first session could be due to the fact that Intentional subjects who used their knowledge more were slower or more variable than those who did not, as they called on their explicit knowledge to guide their responding. This would result in their receiving longer and more variable inter-stimulus intervals, factors that are known to hurt the learning and/or performance of sequences (Stadler 1995; Willingham et al. 1997; Soetens et al. 2004; J.H. Howard Jr., D.V. Howard, N.A. Dennis, and $\mathrm{H}$. Yankovich, in prep.). However, this explanation cannot account for the negative correlation seen here. Overall reaction time on the second epoch did not correlate significantly with implicit learning on epoch 3 in either group [Intentional: $r(10)=0.33, P<0.30$; Incidental: $r(10)=-0.25, P<0.43$ ]. (No such correlations were found with overall speed for any or all sessions with implicit learning measures from any or all Probe epochs). Further, overall within-subject variability in reaction time (measured by standard deviations for reaction time in the second epoch) also did not correlate with implicit learning on epoch 3 in either group [Intentional: $r(10)=-0.06, P<0.85$; Incidental: $r(10)=-0.20, P<0.54]$. (No such correlations were found for within-subject variability for any or all sessions with implicit learning measures for any or all Probe epochs). Therefore, in the Intentional subjects, it was the use of explicit knowledge, and not the presence of the knowledge itself or the overall speed or variability of responding, that was accompanied by decreased expression of implicit learning on the reaction time measures on the first Probe epoch.

\section{Discussion}

\section{Summary of findings}

These results show that the Explicit/Implicit version of the ASRT introduced here is useful for studying the interaction between explicit knowledge and implicit sequence learning. This is so because people with and without explicit knowledge can be distinguished clearly. That is, people given Incidental instructions were unable to explicitly discover any pattern, whether this was assessed by responses to questionnaires or by performance on generation and card sorting tasks. In contrast, those given Intentional instructions were able to gain full explicit knowledge of the pattern on Cued epochs as assessed by sequence description and these same measures. The Intentional subjects were also able to use this knowledge on Cued blocks, as demonstrated by their ability to speed their responses to Pattern-High versus RandomHigh trials, unlike Incidental subjects. Furthermore, for those who do have explicit knowledge, implicit learning can be measured on the Probe trials. That is, Probe blocks enabled us to measure implicit sequence learning in Intentional subjects who have explicit knowledge of the pattern, but do not know that it is relevant without the cues.

From the reaction time and accuracy measures, three main findings on explicit/implicit interactions emerged. First, Probe blocks revealed that implicit learning, as measured by both the accuracy and reaction time triplet type effects, occurred to the same degree continuously across the sessions with or without explicit knowledge. This was revealed by the fact that Intentional and Incidental groups showed learning to the same extent on Probe epochs.

Second, correlations within the Intentional group suggested that the use of explicit knowledge could affect the expression of implicit sequence learning in the initial Probe epoch. This effect was not seen in subsequent epochs.

Third, reaction time and accuracy were found to behave differently during Cued blocks, showing that they were influenced differently by explicit knowledge. As most SRTT studies look only at end points of learning, and use only reaction time

Explicit and Implicit Interaction in First Session

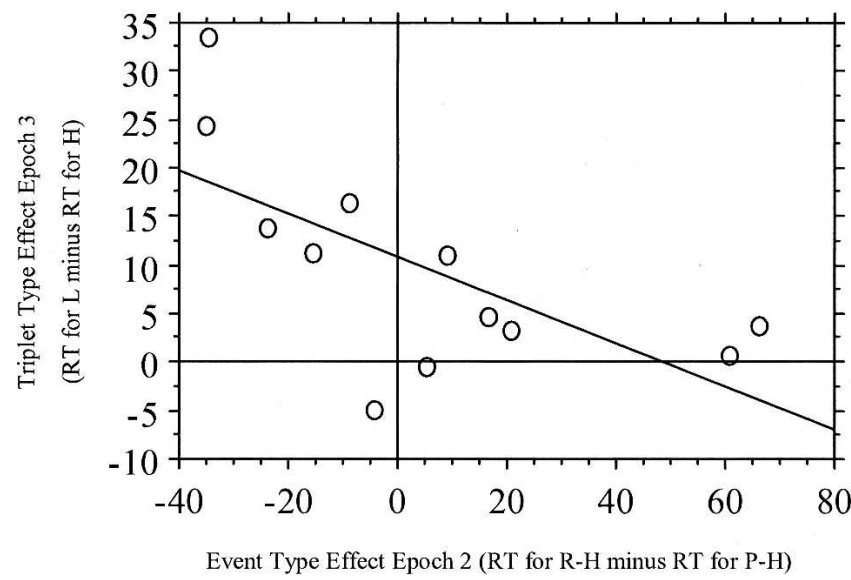

Figure 7. The use of explicit knowledge interferes with implicit learning measures early in learning. The extent to which the person used explicit knowledge was assessed via the Random-High minus Pattern-High Reaction Time difference in the second epoch of a given session, which was Cued. The amount of implicit learning was assessed via the Lowfrequency-High-frequency Reaction Time difference in the third epoch of a given session, which was a Probe epoch. For the first session (as shown here), this correlation was negative and significant, $r(10)=-66$, $p<0.02$, indicating that the more Intentional subjects used their explicit knowledge during cued epoch 2, the less implicit learning they showed on probe epoch 3 . This effect disappeared by the second and third sessions (data not shown); for the second session, $r(10)=0.25, p<0.44$, and for the third session, $r(10)=0.26, p<0.41)$. When we performed these correlations in the Incidental group, they were not significant for any of the sessions (data not shown).

\section{Learning \& Memory}


measures, this finding provides additional insight into sequence learning that has mainly been addressed in the non-human primate literature (Hikosaka et al. 1995, 2002; Miyachi et al. 1997; Rand et al. 2000; Watanabe et al. 2006).

\section{Relation of implicit learning to explicit knowledge}

Findings on the Probe blocks offer insight concerning the controversy of explicit/implicit interactions. Our findings for the most part support the COBALT theory of parallel processes proposed by Willingham et al. (Willingham and Goedert-Eschmann 1999; Willingham 2001; Willingham et al. 2002), which states that implicit sequence learning occurs even when there is also explicit awareness. Probe blocks also revealed that implicit learning increases steadily with practice, irrespective of explicit awareness, and that the amount of implicit learning was identical in the probe blocks, irrespective of explicit knowledge. Thus, it would seem that implicit learning occurs continuously and simultaneously, largely independent of explicit awareness, with the time course unaffected by explicit knowledge. This unaffected time course is in line with other studies of explicit/implicit motor processes such as visuomotor adaptation (Mazzoni and Krakauer 2006), mirror drawing (Gabrieli et al. 1993), rotor pursuit (Tranel et al. 1994), and force field learning (Shadmehr et al. 1998).

Correlation analyses within the Intentional group show, however, an early interaction. In the first session, subjects who used their explicit knowledge more on the Cued epoch had lower implicit learning scores on the reaction time measure on the following Probe epoch. This finding could not be explained by differences in overall speed, or within-subject variability of responses, or even by the time at which explicit awareness was gained. It was specifically the use of explicit knowledge to speed Pattern over Random sequences that was related to implicit learning measures during the first Probe epoch. This relationship disappeared by the second and third sessions, even though Intentional subjects were still using their explicit knowledge to the same extent on the Cued epochs.

Since by the second and third Probe epochs this correlation disappears, and implicit sequence learning is equal in the two groups, it is not plausible to conclude that less implicit learning occurred early in training for the Intentional learners. It is more likely that the extent to which our Intentional learners used explicit knowledge influenced the extent to which they expressed their implicit learning. A recent paper by Jimenez et al. (2006) showed that when Intentional learners encountered a block of control trials in which their explicit knowledge was suddenly no longer relevant, they no longer expressed learning of the embedded trained sequences, even though Incidental learners still did. The investigators interpreted this to mean that a switch from the "explicit mode" can interfere with the expression of implicit learning. In our study, the use of explicit knowledge can be thought of as reflecting more of an explicit mode. The Jimenez et al. explanation suggests that the more subjects used their explicit knowledge during the first Cued blocks, then the more their expression of implicit learning was impaired by the switch to the first Probe block in which their explicit knowledge was suddenly no longer useful due to the removal of the color cues.

This interpretation that explicit knowledge influences the expression, but not the acquisition, of implicit learning has implications for earlier studies showing that explicit learning decreases measures of implicit learning; it might be that the expression of learning was affected, not the implicit learning itself. For example, Fletcher et al. (2004) found that, in young subjects, explicit search completely blocked the expression of implicit learning in a subsequent test block. This interaction was ex- plained as reflecting competition for frontal lobe resources. It may be at this level of performance (frontal lobe) that interference is seen, and with expression but not acquisition. This would be consistent with findings that interference by explicit search has been found in populations with limited frontal lobe resources, such as with aging (Howard and Howard 2001), with stroke (Boyd and Winstein 2006), and with OCD (Kathmann et al. 2005). A transcranial magnetic stimulation (TMS) study showed that the prefrontal cortex is not necessary for sequence learning, in that sequence learning to symbolic cues was not affected by disruption of the dorsolateral prefrontal cortex. TMS to this region, however, did abolish any evidence of sequence learning to spatial cues, again suggesting that the expression but not acquisition of sequence learning is a function of the prefrontal cortex (Robertson et al. 2001). Other explanations, however, are plausible, such as one of competing attentional systems, an explanation in keeping with the dual task literature (Schvaneveldt and Gomez 1998; Jimenez and Mendez 1999).

Regardless of the reason for the negative correlation we found between use of explicit knowledge and the expression of implicit learning early in training, our results indicate that implicit motor sequence learning occurs independently of explicit knowledge.

\section{Reaction time/accuracy dissociation}

Reaction time and accuracy were shown to be differently affected by explicit knowledge in the present study. Unlike the SRTT task, the higher number of errors on the ASRT and other probabilistic sequence learning tasks allows for accuracy to be a sensitive measure of sequence learning. In the present study, accuracy was not affected by explicit knowledge, even in Cued blocks in which explicit knowledge could be used. Because accuracy triplet type effects increased similarly in the two groups across Cued epochs, it appears that, in this particular paradigm, accuracy reflects only implicit learning, whereas reaction time also incorporated aspects of explicit knowledge and strategy.

Several reasons may account for this dissociation. Previous research indicates that sequence learning affects accuracy measures due, at least in part, to anticipatory errors in which subjects make responses consistent with the regularity to Low-frequency events that are inconsistent (Howard et al. 2004a). Anticipatory errors reflecting explicit knowledge cannot occur in the present task, because the sequence is never violated on Pattern trials during Cued blocks. However, there are other sources of errors on all trials; these include general "oops" errors and anticipatory errors due to implicit learning. If explicit knowledge could be used to increase accuracy on Pattern trials by decreasing these other types of errors, then we would expect explicit awareness to increase Pattern-High accuracy over Random-High accuracy for the Intentional participants. This is not the case (see Fig. 6), even though accuracy on High-frequency trials asymptotes at $~ 95 \%$. This lack of effect of explicit knowledge on accuracy suggests either that a ceiling effect is occurring, or that explicit awareness cannot be used to improve accuracy in this paradigm. In contrast, explicit awareness can be used to speed reaction times to these same trials.

Another reason for this accuracy/reaction time dissociation may be that accuracy and reaction time reflect different learning mechanisms. Dissociations between speed and accuracy have been found in non-human primate studies of sequence learning. Using a $2 \times 5$ sequence learning task, errors and reaction time were found to show different behavioral characteristics for transfer. Learning as measured by error rate transferred to the opposite hand, whereas learning measured by reaction time did not (Rand et al. 2000). Error and reaction time also showed different retention characteristics where the interfering effects of different se- 
quences affected accuracy but not speed (Hikosaka et al. 2002). It was proposed that two separate mechanisms of learning occurred, one that affected accuracy and the other that affected speed. Interestingly, a study using the $2 \times 10$ task in humans (Watanabe et al. 2006) found that explicit knowledge transfer affected accuracy but not reaction time measures, which is consistent with our finding in showing a dissociation, but inconsistent in showing reversed effects from ours.

Some additional insight into reaction time/accuracy dissociations may be revealed by an ERP study with humans conducted by Russeler and Rosler (2000). In this study, subjects learned sequences either explicitly or implicitly, and were then subjected to motor sequence deviants or perceptual sequence deviants. Both instruction groups responded in the same way to motor deviants, in that both groups showed reaction time and accuracy costs. In contrast, the two groups and the measures were differentially affected by perceptual deviants, such that the explicit group showed reaction time but not accuracy costs, and the implicit group showed no costs at all. This suggests that implicit sequence learning is highly motor-dependent, with accuracy measures reflecting motor-driven sequence learning, but, in contrast, reaction time incorporates perceptual and strategic as well as motor-driven aspects of sequence learning.

Dual task studies using probabilistic paradigms have also shown reaction time and accuracy dissociations, although no explicit awareness was gained (Schvaneveldt and Gomez 1998; Jimenez and Mendez 1999). Therefore, it may not be explicit awareness per se, but simply competing attentional systems that may affect one but not the other measure of learning.

The particular dissociation seen in our paradigm can also be interpreted in light of Willingham's (1999) proposal that motor skill learning consists of four processes, strategic, perceptual motor integration, sequence learning, and dynamic learning, and two modes, explicit and implicit. It is evident from our data that in the present task, reaction time is affected by mode, but accuracy is not. On Cued blocks when explicit knowledge could be used, reaction time differences between Pattern-High- and Random-High-frequency trials reflect how explicit knowledge can affect reaction time. The large variability in the Intentional group's RT triplet type effects on Cued epochs also likely reflects the effects of strategy, with different people adopting different strategies.

The Explicit/Implicit ASRT task reveals both qualitative and quantitative differences between explicit and implicit sequence learning. In contrast, the SRTT generally can distinguish between the two only by faster performance for explicit learners, although qualitative differences have been revealed by different factors such as context (Jimenez et al. 2006). The presence of Probe epochs and the availability and continuous nature of both accuracy and reaction time measures make the Explicit/Implicit ASRT useful for studying various aspects of explicit and implicit sequence learning. In addition, the Accuracy/RT dissociation is useful in that, oftentimes, deficits in certain populations are found in one measure but not the other. For example, age deficits are most clearly seen in accuracy learning measures (Howard et al. 2004a). The same was the case for schizophrenics versus normal controls (Schwartz et al. 2003). In addition, the present paradigm may be useful for neuroimaging studies, as concurrent explicit/implicit learning paradigms increasingly have been used to reveal deficits in frontal-striatal recruitment for certain populations such as older adults (Howard et al. 2004b; Aizenstein et al. 2006).

In the present study, it was revealed that implicit and explicit learning occurred simultaneously, although correlations early in training suggest that an explicit mode may interfere with the expression of implicit learning. Such explicit/implicit interactions may be more pronounced in populations with impaired frontal-striatal circuitry (Howard and Howard 2001; Kathmann et al. 2005; Boyd and Winstein 2006). Our findings elucidate the reasons for the mixed results seen in the sequence learning literature when exploring explicit/implicit interactions, and highlight the conclusion that implicit learning can occur simultaneously with, and independent of, explicit knowledge.

\section{Materials and Methods}

\section{Participants}

Twenty-four right-handed volunteers (aged 19 to $21 \mathrm{yr}$, two males and 22 females) were recruited from Georgetown University to participate in the study for credit. All subjects signed informed consent and were tested between 4:00 p.m. and 5:30 p.m.

\section{Alternating Serial Reaction Time (ASRT) task}

Participants responded to a filled-in circle that appeared in one of four open circles arranged horizontally on a computer screen by pressing one of four corresponding keys on the computer keyboard. The circle remained filled in until participants pressed the correct key, at which time it disappeared and another target appeared after a delay of $120 \mathrm{msec}$. By giving feedback at the end of each block to focus more on accuracy or on speed, subjects were guided to $92 \%$ overall accuracy.

All participants completed three sessions of the ASRT in a single lab visit, with each session taking $\sim 20 \mathrm{~min}$. In each session, there were three epochs, with five blocks in each epoch. Each block began with five Random black trials (for warm-up) and was followed by 80 experimental trials. These 80 trials consisted of an eight-item sequence, in which Pattern trials alternated with Random trials (e.g., 1r3r4r2r), and this eight-item sequence repeated 10 times in a block. Subjects received one of five possible sequences (1r2r3r4r was excluded due to possible ease of discovery). On Random trials, any one of the four positions occurred.

In the first two epochs of each session (Cued epochs), Pattern trials were cued by color (gray or red), whereas the alternating Random trials had black targets. For the last epoch in each session (Probe epoch), all trials had black targets. Thus, epochs 1, 2, $4,5,7$, and 8 were color Cued with black targets (Random) alternating with colored targets (Pattern), whereas epochs 3, 6, and 9 were Probe epochs consisting of all black targets.

Half of the subjects were given Intentional instructions and half were given Incidental instructions. The Incidental group was not told about the regularity, and the alternating colors on the Cued epochs were explained as being able to "help participants distinguish between trials." The Intentional group was alerted to the regularity by the addition of the following directions: "Black targets always have randomly chosen locations. However, gray (or for those given red cues, "red") targets always follow a pattern. The pattern of the gray targets repeats every four gray targets. . . In all blocks, in both sessions, the gray targets will follow the same four-sequence pattern." Neither group was alerted to the regularity in the Probe blocks, although this regularity was identical to the one in the Cued blocks.

\section{Tests of explicit knowledge}

The following sequence description task was given only to the Intentional group. After each training block, subjects were asked to verbally report the four-sequence pattern the cued stimuli had followed. The four positions corresponded to numbers 1 through 4 , and subjects were required to guess after each block. Subjects also gave a confidence rating between 1 and 5, with 5 being the most confident.

Then, at the end of the ASRT testing, both instruction groups received the following tests of explicit knowledge. First, they were given a generation task in which they were asked to produce a typical 16-unit-long pattern that had occurred during each session. They did so by writing out a typical sequence in numbers into 16 blank spaces. They were instructed to include 
both gray and black targets and the four positions corresponded to numbers 1 through 4 .

Next, subjects in both instruction groups were given interviews that asked increasingly specific questions regarding their knowledge of any regularity in the sequences they had encountered in the alternating color Cued blocks and in the all black Probe blocks.

Last, they were given a card sorting task for probe epochs. For this task, participants were given a stack of 64 index cards, each of which contained three rows of four circles. One circle on each row was filled with a black color. Each line represented one trial, so each card denoted three consecutive trials on the ASRT task (a "triplet"). Participants were told to sort each card into one of two piles labeled "Occurred Most Often" and "Occurred Least Often" based on how often they thought these triplets of trials occurred in the Probe epochs (Japikse et al. 2001).

\section{Acknowledgments}

This work was supported by NIH Grants R37AG15450 and HD40095.

\section{References}

Aizenstein, H.J., Butters, M.A., Clark, K.A., Figurski, J.L., Andrew Stenger, V., Nebes, R.D., Reynolds III, C.F., and Carter, C.S. 2006. Prefrontal and striatal activation in elderly subjects during concurrent implicit and explicit sequence learning. Neurobiol. Aging 27: 741-751.

Ashe, J., Lungu, O.V., Basford, A.T., and Lu, X. 2006. Cortical control of motor sequences. Curr. Opin. Neurobiol. 16: 213-221.

Barone, P. and Joseph, J.P. 1989. Prefrontal cortex and spatial sequencing in macaque monkey. Exp. Brain Res. 78: 447-464.

Boyd, L. and Winstein, C. 2006. Explicit information interferes with implicit motor learning of both continuous and discrete movement tasks after stroke. J. Neurol. Phys. Ther. 30: 46-57.

Cohen, A., Ivry, R.I., and Keele, S.W. 1990. Attention and structure in sequence learning. J. Exp. Psychol. Learn. Mem. Cognit. 16: 17-30.

Curran, T. and Keele, S.W. 1993. Attentional and nonattentional forms of sequence learning. J. Exp. Psychol. Learn. Mem. Cognit. 19: 189-202.

Destrebecqz, A., Peigneux, P., Laureys, S., Degueldre, C., Del Fiore, G., Aerts, J., Luxen, A., Van Der Linden, M., Cleeremans, A., and Maquet, P. 2005. The neural correlates of implicit and explicit sequence learning: Interacting networks revealed by the process dissociation procedure. Learn. Mem. 12: 480-490.

Exner, C., Koschack, J., and Irle, E. 2002. The differential role of premotor frontal cortex and basal ganglia in motor sequence learning: Evidence from focal basal ganglia lesions. Learn. Mem. 9: 376-386.

Fletcher, P.C., Zafiris, O., Frith, C.D., Honey, R.A.E., Corlett, P.R., Zilles, K., and Fink, G.R. 2004. On the benefits of not trying: Brain activity and connectivity reflecting the interactions of explicit and implicit sequence learning. Cereb. Cortex 15: 1002-1015.

Frensch, P.A. 1998. One concept, multiple meanings: On how to define the concept of implicit learning. In Handbook of implicit learning (eds. M.A. Stadler and P.A. Frensch), pp. 47-104. Sage Publications, Inc., Thousand Oaks, CA.

Frensch, P.A., Buchner, A., and Lin, J. 1994. Implicit learning of unique and ambiguous serial transitions in the presence and absence of a distractor task. J. Exp. Psychol. Learn. Mem. Cognit. 20: 567-584.

Gabrieli, J.D., Corkin, S., Mickel, S.F., and Growdon, J.H. 1993. Intact acquisition and long-term retention of mirror-tracing skill in Alzheimer's disease and in global amnesia. Behav. Neurosci. 107: 899-910.

Grafton, S.T., Hazeltine, E., and Ivry, I. 1995. Functional mapping of sequence learning in normal humans. J. Cognit. Neurosci. 7: 497-510.

Halsband, U. and Lange, R.K. 2006. Motor learning in man: A review of functional and clinical studies. J. Physiol. (Paris) 99: 414-424.

Hazeltine, E., Grafton, S.T., and Ivry, R. 1997. Attention and stimulus characteristics determine the locus of motor-sequence encoding. A PET study. Brain 120: 123-140.

Hikosaka, O., Rand, M.K., Miyachi, S., and Miyashita, K. 1995. Learning of sequential movements in the monkey: Process of learning and retention of memory. J. Neurophysiol. 74: 1652-1661.

Hikosaka, O., Rand, M.K., Nakamura, K., Miyachi, S., Kitaguchi, K., Sakai, K., Lu, X., and Shimo, Y. 2002. Long-term retention of motor skill in macaque monkeys and humans. Exp. Brain Res. 147: 494-504.

Histed, M.H. and Miller, E.K. 2006. Microstimulation of frontal cortex can reorder a remembered spatial sequence. PLoS Biol. 4: e134.
Howard Jr., J.H. and Howard, D.V. 1997. Age differences in implicit learning of higher order dependencies in serial patterns. Psychol. Aging 12: 634-656.

Howard, D.V. and Howard Jr., J.H. 2001. When it does hurt to try: Adult age differences in the effects of instructions on implicit pattern learning. Psychon. Bull. Rev. 8: 798-805.

Howard Jr., J.H., Howard, D.V., Dennis, N.A., Yankovich, H., and Vaidya, C.J. 2004a. Implicit spatial contextual learning in healthy aging. Neuropsychology 18: 124-134.

Howard, D.V., Howard Jr., J.H., Japikse, K., DiYanni, C., Thompson, A. and Somberg, R. 2004b. Implicit sequence learning: Effects of level of structure, adult age, and extended practice. Psychol. Aging 19: 79-92.

Howard Jr., J.H., Howard, D.V., Japikse, K.C., and Eden, G.F. 2006 Dyslexics are impaired on implicit higher-order sequence learning, but not on implicit spatial context learning. Neuropsychologia 44: 1131-1144.

Japikse, K., Howard, D.V., and Howard Jr., J.H. 2001. Evaluation of a direct nonverbal measure of declarative sequence knowledge. $J$. Cognit. Neurosci. Suppl. 12: 62.

Jimenez, L. and Mendez, C. 1999. Which attention is needed for implicit sequence learning? J. Exp. Psychol. Learn. Mem. Cognit. 25: 236-259.

Jimenez, L., Vaquero, J.M., and Lupianez, J. 2006. Qualitative differences between implicit and explicit sequence learning. J. Exp. Psychol. Learn. Mem. Cogn. 32: 475-490.

Kathmann, N., Rupertseder, C., Hauke, W., and Zaudig, M. 2005. Implicit sequence learning in obsessive-compulsive disorder: Further support for the fronto-striatal dysfunction model. Biol. Psychiatry 58: $239-244$.

Mazzoni, P. and Krakauer, J.W. 2006. An implicit plan overrides an explicit strategy during visuomotor adaptation. J. Neurosci. 26: 3642-3645.

Miyachi, S., Hikosaka, O., Miyashita, K., Karadi, Z., and Rand, M.K. 1997. Differential roles of monkey striatum in learning of sequential hand movement. Exp. Brain Res. 115: 1-5.

Negash, S., Petersen, L.E., Geda, Y.E., Knopman, D.S., Boeve, B.F., Smith, G.E., Ivnik, R.J., Howard, D.V., Howard Jr., J.H., and Petersen, R.C. 2006. Effects of ApoE genotype and mild cognitive impairment on implicit learning. Neurobiol. Aging doi:10.1016/j.neurobiolaging. 2006.04.004.

Nissen, M.J. and Bullemer, P. 1987. Attentional requirements of learning: Evidence from performance measures. Cognit. Psychol. 19: $1-32$.

Rand, M.K., Hikosaka, O., Miyachi, S., Lu, X., Nakamura, K., Kitaguchi, K., and Shimo, Y. 2000. Characteristics of sequential movements during early learning period in monkeys. Exp. Brain Res. 131: $293-304$

Rauch, S.L., Savage, C.R., Brown, H.D., Curran, T., Alpert, N.M., Kendrick, A., Fischman, A.J., and Kosslyn, S.M. 1995. A PET investigation of implicit and explicit sequence learning. Hum. Brain Mapp. 3: 271-286.

Rauch, S.L., Whalen, P.J., Savage, C.R., Curran, T., Kendrick, A., Brown, H.D., Bush, G., Breiter, H.C., and Rosen, B.R. 1997. Striatal recruitment during an implicit sequence learning task as measured by functional magnetic resonance imaging. Hum. Brain Mapp. 5: $124-132$.

Robertson, E.M., Tormos, J.M., Maeda, F., and Pascual-Leone, A. 2001. The role of the dorsolateral prefrontal cortex during sequence learning is specific for spatial information. Cereb. Cortex 11: $628-635$.

Russeler, J. and Rosler, F. 2000. Implicit and explicit learning of event sequences: Evidence for distinct coding of perceptual and motor representations. Acta Psychol. 104: 45-67.

Sakai, K., Hikosaka, O., Miyauchi, S., Takino, R., Sasaki, Y., and Putz, B. 1998. Transition of brain activation from frontal to parietal areas in visuomotor sequence learning. J. Neurosci. 18: 1827-1840.

Schvaneveldt, R.W. and Gomez, R.L. 1998. Attention and probabilistic sequence learning. Psychol. Res. 61: 175-190.

Schwartz, B.L., Howard, D.V., Howard Jr., J.H., Hovaguimian, A., and Deutsch, S.I. 2003. Implicit learning of visuospatial sequences in schizophrenia. Neuropsychology 17: 517-533.

Shadmehr, R., Brandt, J., and Corkin, S. 1998. Time-dependent motor memory processes in amnesic subjects. J. Neurophysiol. 80: $1590-1597$.

Shanks, D.R. 2005. Implicit learning. In Handbook of cognition (eds. K. Lamberts and R. Goldstone), pp. 202-220. Sage, London, UK.

Shanks, D.R. and Channon, S. 2002. Effects of a secondary task on "implicit" sequence learning: Learning or performance? Psychol. Res. 66: 99-109.

Smith, J.G. and McDowall, J. 2004. Impaired higher order implicit sequence learning on the verbal version of the serial reaction time 
task in patients with Parkinson's disease. Neuropsychology 18: $679-691$.

Soetens, E., Melis, A., and Notebaert, W. 2004. Sequence learning and sequential effects. Psychol. Res. 69: 124-137.

Stadler, M.A. 1995. Role of attention in implicit learning. J. Exp. Psychol. Learn. Mem. Cognit. 21: 674-685.

Tranel, D., Damasio, A.R., Damasio, H., and Brandt, J.P. 1994. Sensorimotor skill learning in amnesia: Additional evidence for the neural basis of nondeclarative memory. Learn. Mem. 1: 165-179.

Watanabe, K., Ikeda, H., and Hikosaka, O. 2006. Effects of explicit knowledge of workspace rotation in visuomotor sequence learning. Exp. Brain Res. 174: 673-678.

Weickert, T.W., Terrazas, A., Bigelow, L.B., Malley, J.D., Hyde, T., Egan, M.F., Weinberger, D.R., and Goldberg, T.E. 2002. Habit and skill learning in schizophrenia: Evidence of normal striatal processing with abnormal cortical input. Learn. Mem. 9: 430-442.

Willingham, D.B. 1999. The neural basis of motor-skill learning. Curr.
Dir. Psychol. Sci. 8: 178-182.

Willingham, D.B. 2001. Becoming aware of motor skill. Trends Cogn. Sci. 5: $181-182$

Willingham, D.B. and Goedert-Eschmann, K. 1999. The relation between implicit and explicit learning: Evidence for parallel development. Psychol. Sci. 10: 531-534.

Willingham, D.B., Greenberg, A.R., and Thomas, R.C. 1997. Response-to-stimulus interval does not affect implicit motor sequence learning, but does affect performance. Mem. Cognit. 25: 534-542.

Willingham, D.B., Salidis, J., and Gabrieli, J.D.E. 2002. Direct comparison of neural systems mediating conscious and unconscious skill learning. J. Neurophysiol. 88: 1451-1460.

Received September 26, 2006; accepted in revised form January 9, 2007. 


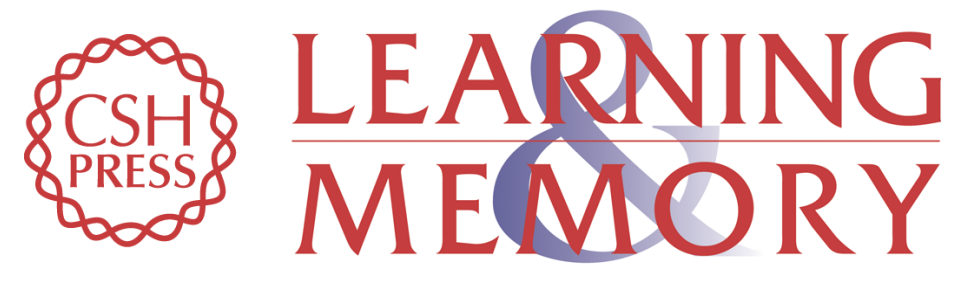

\section{Implicit probabilistic sequence learning is independent of explicit awareness}

Sunbin Song, James H. Howard, Jr. and Darlene V. Howard

Learn. Mem. 2007, 14:

Access the most recent version at doi:10.1101//m.437407

References

This article cites 50 articles, 6 of which can be accessed free at: http://learnmem.cshlp.org/content/14/3/167.full.html\#ref-list-1

License

Email Alerting Service
Receive free email alerts when new articles cite this article - sign up in the box at the top right corner of the article or click here. 